\title{
RESEARCH ON SERVICE PLATFORM OF INTERNET OF THINGS FOR SMART CITY
}

\author{
Wei Wang, Zhonghuan He, Diquan Huang, Xi Zhang \\ Chongqing Institute of Survey ing and Mapping of NASMG, Chongqing, China - wangweigis@126.com
}

Commission IV, WG IV/6

KEY WORDS : Internet of Things, Sensor Networks, Smart City, Sensor

\begin{abstract}
ABS TRACT:
The application of Internet of Things in surveying and mapping industry basically is at the exploration stage, has not formed a unified standard. Chongqing Institute of Surveying and Mapping (CQISM) launched the research project "Research on the Technology of Internet of Things for Smart City". The project focuses on the key technologies of information transmission and exchange on the Internet of Things platform. The data standards of Internet of Things are designed. The real-time acquisition, mass storage and distributed data service of mass sensors are realized. On this basis, CQISM deploys the prototype platform of Internet of Things. The simulation application in Connected Car proves that the platform design is scientific and practical.
\end{abstract}

\section{INTRODUCTION}

With the development and application of digital city, the emergence of new technology and the application of sensor networks, Internet of Things, wireless networks, mobile Internet, cloud computing, Big Data and so on, city is becoming more and more intelligent. Smart City is developing from the concept to the application.

Smart City is the product of accelerated development of the new generation information technology and knowledge-based economy, based on the network combination of the Internet, telecommunications network, broadcast network, wireless broadband network and other networks. Smart City is the inevitable trend from Digital City to Intelligent City. Smart City has the following three features: instrumented, interconnected, intelligent. Digital City and Internet of Things is the two basic of Smart City. The technology of Digital City is already mature and widely used. The future Internet of Things links uniquely identifiable things to their virtual representations in the Internet containing or linking to additional information on their identity, status, location or any other business, social or privately relevant information at a financial or non-financial pay-off that exceeds the efforts of information provisioning and offers information access to non-predefined participants.

As of 2014, research into the Internet of Things is still in its infancy. The application of Internet of Things in surveying and mapping industry basically is at the exploration stage. In consequence, we lack the unified standard for Internet of Things. Chongqing Institute of Surveying and Mapping (CQISM), which is a directly affiliated institution of National Administration of Surveying, Mapping and Geoinformation (NASMG), launched the research project "Research on the Technology of Internet of things for Smart City" in response to the Smart City strategy of NASMG. The project focuses on the key technologies of information transmission and exchange on the Internet of things platform. The data standards of Internet of things are designed. The real-time acquisition, mass storage and distributed data service of mass sensors are realized. On this basis, CQISM deploys the prototype platform of Internet of Things.

\section{PLATFORM ARCHITECTURE}

The prototyping platform of Internet of Things in CQISM is designed for three-tier structure: Data Acquisition Tier, Service Tier and Application Tier, as illustrated in Figure 1. Their functions are as follows:

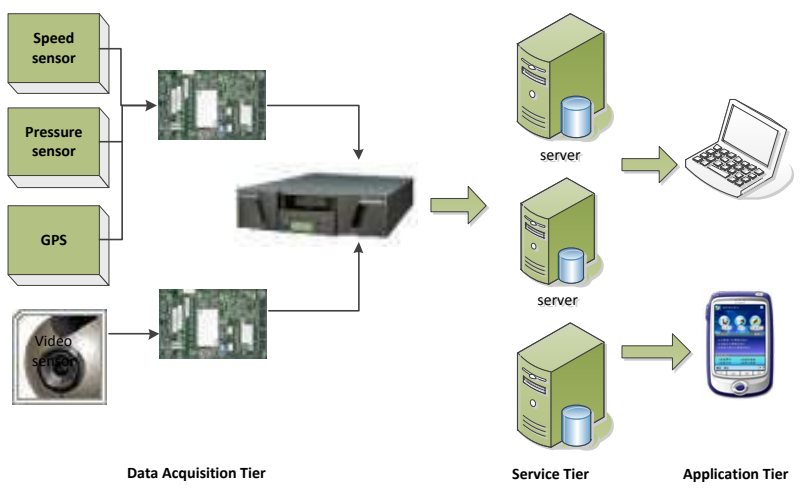

Figure 1. The platform architecture

\subsection{Data Acquisition Tier}

Data Acquisition Tier is the main component of the central control unit. It is responsible for receiving and parsing sensor 
data and sends data. Real-time data is compressed by a custom protocol to the Data Center via a transmission medium.

\subsection{Service Tier}

The Service Tier is constituted of Gateway and Data Center. The function of Gateway is to receive and analyse real-time distributed multi-source sensor data. The Data Center is responsible for classifying and storing real-time data which manages service distribution.

\subsection{Application Tier}

The Application Tier is to perform real-time status information of the sensors in a variety of ways.

\section{DEVELOPMENT APPROACHES}

The core of the Data Acquisition Tier is the Central Control Unit (CCU). CCU applies JAVA technology, completing the conversion from hardware signal to the sensor information. In this process, using the signal techniques converts an electric signal to serial data output, as the serial data is parsed to extract the sensor data. Finally, sensor data is merged and data records are standardized. According to industry standards, the sensor data is compressed to distribution by way of the Internet. In summary, CCU transfers the electrical signal to the sensor useful information by information analysis \& fusion and storages sensor information (Figure 2).

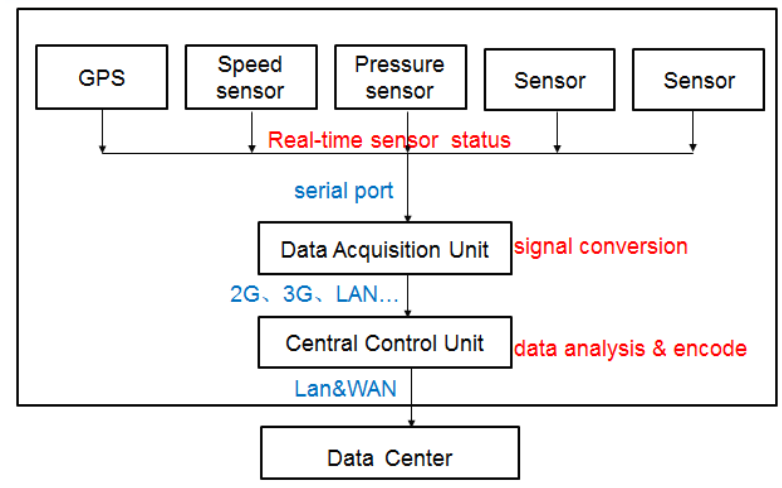

Figure 2. The Data flow of Data Acquisition Tier

The Service Tier consists of three modules: Information Gateway, Data Center, Video Server. Information Gateway is developed based on Java MINA, with logical operators fast, large number of concurrent and running stable. Information Gateway establishes a connection with the CCU through the TCP/IP network, and verifies the legitimacy of CCU according to custom specifications, and parses the sensor data records which uploaded by $\mathrm{CCU}$, removing the error message, and extract the active sensor information, giving CCU feedback, and finally sends information to the Data Center. The Data Center is developed based on oracle database. The Data Center storages, analyses and services the real-time sensor data which is uploaded from Information Gateway. Video server is developed based on HikVision SDK, consisting of the storage servers, streaming media servers. Video server consists of a video management system which is developed by Microsoft technology for video data storage, analysis, display and so on. It achieves the management of user information and permissions, monitors real-time preview and historical point of playback.

The Application Tier which is developed using advanced FLEX web technology, and the geographic information system platform ArcGIS application interface, as construction with $\mathrm{B} / \mathrm{S}$ architecture. The Application Tier achieves sensor data stream from the Data Center to the client to show. It obtains real-time sensor data by use of network services and presents real-time sensor information by dynamic network technology.

\section{EXPERIMENT}

In order to validate the feasibility of prototype platform, we design the simulation experiment in Connected Car. We assume that the vehicles are equipped with various sensors and other devices for the determination of the actual condition of the vehicles themself, such as speed, pressure and GPS, etc. Through the Internet of things platform, the application manages the information of the vehicles and all sensors. And the application displays all real-time information to the user through various forms (Figure 3).

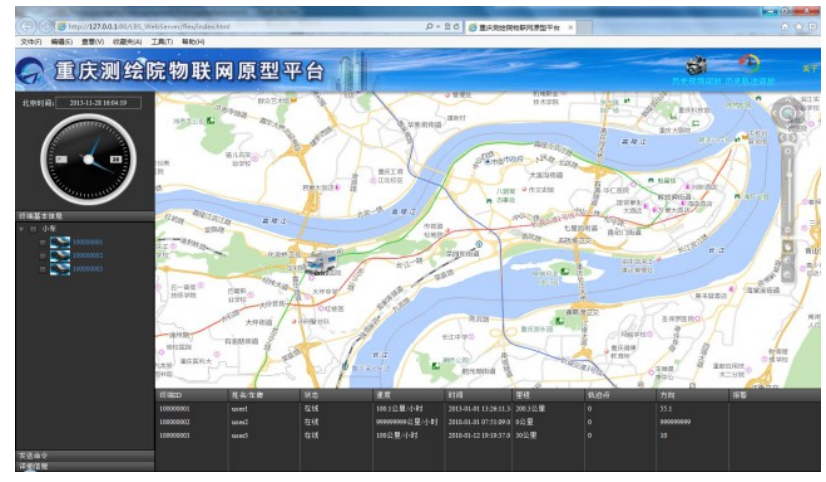

Figure 3. The application of Connected Car

The application is developed based on $\mathrm{B} / \mathrm{S}$ architecture, the FlexViewer 3 framework and ArcGIS API for Flex 3. Thus users can use the application through the browser like IE, Firefox and Chrome, etc.

The major functions of the application are as follows:

1. The basic functions of traditional GIS.

2. Display the basic information of the vehicles (Figure 3).

3. Represent the real-time information of sensors through different ways such as text, number and dashboard (Figure 4), etc.

4. Analyse and represent the historical data. Figure 5 is the real-time analysis of speed data. Figure 6 is the reappearance of GPS historical track data.

From the experiment, we see that the platform design is practical, all functions of the application are easy to operate, and the user experience is rich. 


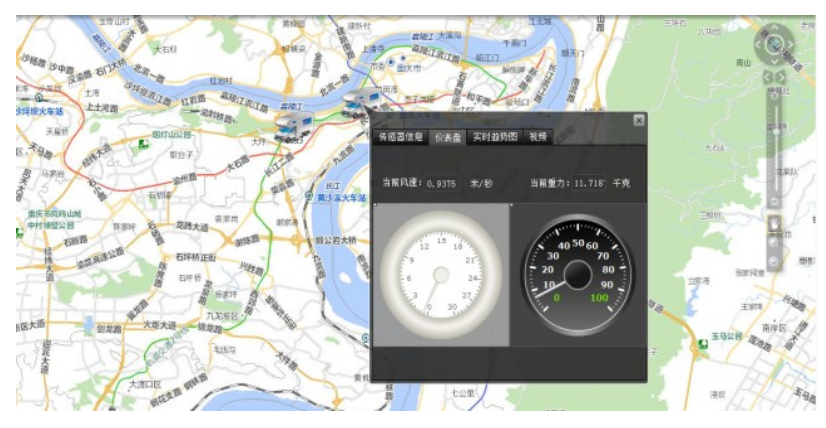

Figure 4. The application of Connected Car

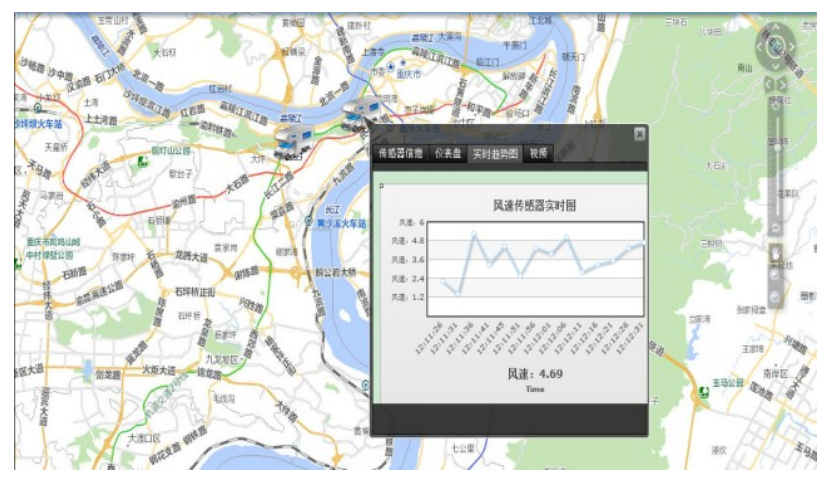

Figure 5. The analy sis of real-time data

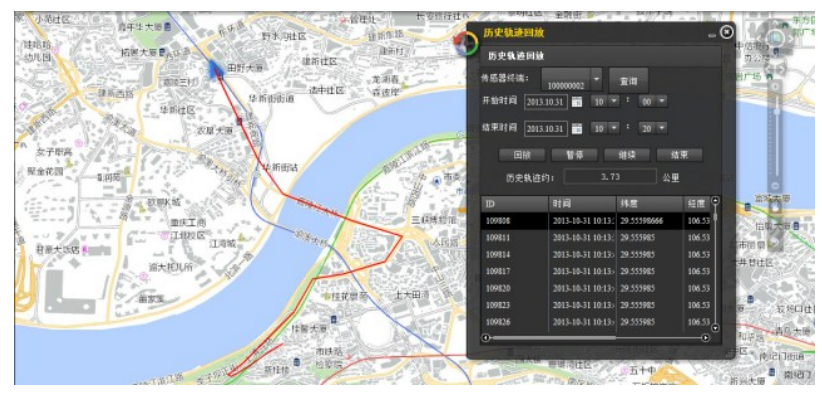

Figure 6. The reappearance of historical data

\section{CONCLUTIONS AND FUTURE WORKS}

The service platform of Internet of Things in CQISM focuses on the key technologies of information transmission and exchange. The data standards of Internet of things are designed. The real-time acquisition, mass storage and distribution data service of mass sensors are realized. The platform design owns high performance, high availability and high scalability, which can be applied in Connected Car, the prevention of geological hazards and environmental monitoring by extending different types of sensors. The application in Connected Car proves that the platform design is scientific and practical. In the future, the Data Center will be extended to Cloud Computing Data Center and Cloud Services.

\section{ACKNOWLEDGEMENTS}

The work is supported by the National Administration of Surveying, Mapping and Geoinformation under grant
"Demonstration Base Construction of Information-based Mapping System in CQISM" and "Research on Key Technologies and Applications of Internet of Things for Smart City".

\section{REFERENCES}

Aberer, K., Hauswirth, M., and A. Salehi, M., 2006. The Global Sensor Networks middleware for efficient and flexible deployment and interconnection of sensor networks, Tech. Rep. LSIR-REPORT-2006-006, Ecole Polytechnique Fédérale de Lausanne.

Artz, M., 2013. The New Age of Real-Time GIS. ESRI. http://blogs.esri.com/esri/esri-insider/2013/04/01/thenew-age-ofreal-time-gis/ (1 May, 2013).

Conti, J., 2006. The Internet of things. Communications Engineer. (6), pp. 20

Gong, J., et al., 2010. Multi-source geospatial information integration and sharing in Virtual Globes, Science China Technological Sciences, (53), pp. 1-6.

Laurini, R., Servigne, S.,Noel, G., 2005. Soft Real-Time GIS for Disaster Monitoring. In Geo-information for Disaster Management, eds. P. Oosterom, S. Zlatanova \& E. Fendel. Springer Berlin Heidelberg, pp. 465-479.

Yang, C., et al., 2009. Introduction to distributed geographic information processing research. International Journal of Geographical Information Science, 23(5), pp. 553-560. 